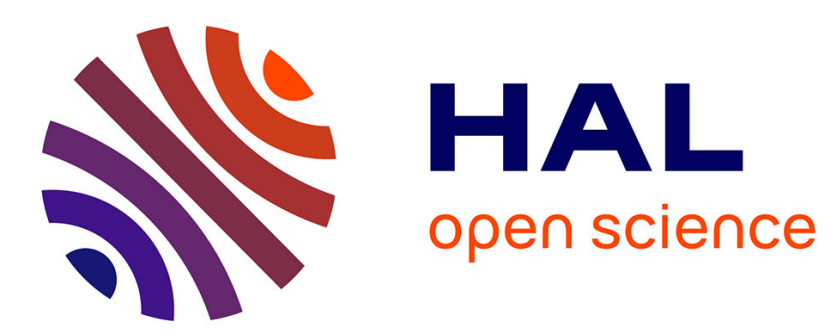

\title{
EVIDENCE FOR AND EXPLICATION OF THE D-STATE IN FEW-NUCLEON SYSTEMS
}

\author{
D. Lehman
}

\section{To cite this version:}

D. Lehman. EVIDENCE FOR AND EXPLICATION OF THE D-STATE IN FEW-NUCLEON SYSTEMS. Journal de Physique Colloques, 1990, 51 (C6), pp.C6-47-C6-60. 10.1051/jphyscol:1990604 . jpa-00230867

\section{HAL Id: jpa-00230867 https://hal.science/jpa-00230867}

Submitted on 1 Jan 1990

HAL is a multi-disciplinary open access archive for the deposit and dissemination of scientific research documents, whether they are published or not. The documents may come from teaching and research institutions in France or abroad, or from public or private research centers.
L'archive ouverte pluridisciplinaire HAL, est destinée au dépôt et à la diffusion de documents scientifiques de niveau recherche, publiés ou non, émanant des établissements d'enseignement et de recherche français ou étrangers, des laboratoires publics ou privés. 
EVIDENCE FOR AND EXPLICATION OF THE D-STATE IN FEW-NUCLEON SYSTEMS

\author{
D.R. LEHMAN
}

Department of Physics, The George Washington University, Washington, DC 20052, U.S.A.

\begin{abstract}
Résumé - Une revue de notre compréhension actuelle des propriétés de l'état $\mathrm{D}$ dans les systèmes à peu de nucléons, ${ }^{2} \mathrm{H}$, ${ }^{3} \mathrm{H},{ }^{3} \mathrm{He},{ }^{4} \mathrm{He}$, et ${ }^{6} \mathrm{Li}$, est présentée. Nous soulignons l'interprétation théorique des dernières évidences expérimentales, en particulier celles obtenues avec des faisceaux polarisés. Nous essayons de donner une présentation théorique cohérente de notre compréhension des constantes de normalisation de la fonction d'onde asymptotique de l'état $\mathrm{D}$ pour les systèmes ci-dessus. Le but est de relier les prédictions théoriques du rapport des normalisations des fonctions d'onde asymptotiques de l'état $D$ et de l'état $S$ à la présence de la force tensorielle entre deux nucléons. Nous présentons ensuite ce qui peut être appris, à partir des réactions de capture radiative, concernant l'état $\mathrm{D}$ dans les noyaux à peu de nucléons considérés, Nous concluons par un résumé de ce que nous savons aujourd'hui de l'état D dans les systèmes à peu de nucléons et nous proposons un développement possible de recherches expérimentales et théoriques.

Abstract - A review is given of our current understanding of the D-state properties of the few-nucleon nuclei ${ }^{2} \mathrm{H},{ }^{3} \mathrm{H}$, ${ }^{3} \mathrm{He},{ }^{4} \mathrm{He}$, and ${ }^{6} \mathrm{Li}$. Emphasis is placed on the theoretical interpretation of the latest empirical evidence, especially those experiments involving polarized beams. An attempt is made to provide a coherent theoretical overview of our current understanding of the D-wave asymptotic normalization constants of the above few-nucleon systems. The aim is to tie the theoretical predictions of the ratio of the $\mathrm{D}$-wave to $\mathrm{S}$-wave asymptotic normalization constants to the presence of the two-nucleon tensor force. Following this discussion is an assessment of what has been learned about the D-state in the above few-nucleon nuclei from radiative capture reactions. The review closes with a summary of our current understanding of the D-state in few-nucleon systems and a projection of possible future directions.
\end{abstract}

\title{
1 - INTRODUCTION
}

The existence of a nonzero quadrupole moment for the deuteron and, as a consequence, the presence of a tensor component in the two-nucleon interaction, has been known now for approximately 50 years $/ 1,2 /$. Nevertheless, it has only been in the last decade that a deep understanding has been obtained of the implications of a D-state component in the deuteron wave function. In particular, it has been learned that the percentage of this component in the wave function is not an observable $/ 3,4 /$ and that the key observable is $\eta$, the ratio of the D-wave to S-wave asymptotic normalization constants. It has been shown that there is a very tight linear relationship between the deuteron quadrupole moment (divided by the S-wave asymptotic normalization constant squared) and $\eta$. More importantly, the role of the tensor force, in particular, the one-pion exchange (OPE) component of the tensor force, in determining $\eta$ is understood quite well $/ 5,6 /$. Experimentally, the deuteron quadrupole moment is well determined $/ 7 /$, whereas the experimental value of $\eta$ remains controversial as we will discuss below. Of course, it would be beneficial if we could have an equal understanding of the role of the tensor force in other few-nucleon systems. Naturally, questions concerning the two-nucleon tensor force in other systems were ask early on. Gerjuoy and Schwinger $/ 8 /$ soon realized that the two-nucleon tensor force plays a role in binding the three- and fournucleon nuclei, though it is now known that it does not play as large a role as in the deuteron $/ 9 /$. In the last ten years, exact three-body calculations have produced values for the ${ }^{3} \mathrm{H}$ and ${ }^{3} \mathrm{He} \eta$ 's based on the the most current phenomenological two-nucleon interactions. Evidence exists that there is a linear correlation between the three-body $\eta$ 's and the $\eta$ of the deuteron $\left(\eta_{d}\right)$. Progress has also been made experimentally in determining the three-body $\eta$ 's as well as the closely related distorted-wave-Born-approximation (DWBA) parameter $\mathrm{D}_{2}$. The same cannot be said for ${ }^{4} \mathrm{He}$ and ${ }^{6} \mathrm{Li}$. In fact, it could be said that the work on these systems is still in its infancy. For ${ }^{4} \mathrm{He}$, the calculations are difficult owing to the four-body nature of the problem, whereas for ${ }^{6} \mathrm{Li}$, calculations exist for the alpha-deuteron ( $\alpha$-d) component, but not for the ${ }^{3} \mathrm{H}-{ }^{3} \mathrm{He}$ component. In addition, experimental determinations of the D-state parameters are beset with difficulties not present in the case of the three-body nuclei. On the other hand, radiative capture reactions with polarized deuterons have shown unequivocally the presence of the D-state component in the ${ }^{4} \mathrm{He}$ nucleus. To date, experimental determination of the ${ }^{6} \mathrm{Li} D-$ state parameters is open. All these aspects are very completely covered in detail in two recent reviews $/ 10,11 /$.

In light of the two current review articles $/ 10,11 /$, my purpose is to give a broad overview of the main aspects of current work, update the reviews where appropriate, and attempt to present our current physical understanding of the D-state structure of the few-body nuclei through $A=6$. I will begin with a presentation of the formalism, and then discuss the 'sshell' nuclei, ${ }^{2} \mathrm{H},{ }^{3} \mathrm{H},{ }^{3} \mathrm{He}$, and ${ }^{4} \mathrm{He}$. In each case, I shall begin with our current theoretical understanding followed by a discussion of the most recent experimental results. Then, I shall move from the ' $\mathrm{s}$-shell' to ${ }^{6} \mathrm{Li}$. This will be followed by a discussion of the role of radiative capture reactions in the determination of D-state properties. A brief synopsis at the end will also provide projections for future work.

\section{2- FORMALISM AND DEFINITIONS}

Asymptotic normalization constants are defined for the separation of a nucleus into two fragments, each of which is bound (or considered elementary, e.g., a nucleon), i.e., $A \rightarrow x+y$. If the two fragments have internal coordinates designated by $\mathbf{R}_{x}$ 
and $\mathbf{R}_{\mathrm{y}}$, and charges $\mathrm{Z}_{\mathrm{x}}$ and $\mathrm{Z}_{\mathrm{y}}$, respectively, then as the magnitude of the vector $\rho$ that connects their centers of mass goes to infinity, while $\left|\mathbf{R}_{\mathrm{x}}\right|$ and $\left|\mathbf{R}_{\mathbf{y}}\right|$ remain finite, the wave function of the nucleus $A$ can be written in terms of the asymptotic normalization constants, $C_{l}^{C}$, as follows:

$$
\begin{aligned}
\lim _{\rho \rightarrow \infty} \Psi_{M}^{[J]}(\boldsymbol{\rho}, & \left.\mathbf{R}_{x}, \mathbf{R}_{y}\right) \Rightarrow \sum_{\substack{l \neq=0 \\
l \neq 1}}^{2} C_{l}^{C} N_{W} \frac{W_{-\kappa, l+1 / 2}\left(2 \mu^{C} \rho\right)}{\rho} \\
& \left.\times i^{l} \sum_{J^{\prime}} \sqrt{4 \pi}\left[Y^{[l]}(\hat{\rho}) \times\left[\varphi^{\left[j_{x}\right]}\left(\mathbf{R}_{x}\right) \times \psi^{\left[j_{y}\right]}\left(\mathbf{R}_{y}\right)\right]^{\left[J^{\prime}\right]}\right]\right]_{M}^{[J]}
\end{aligned}
$$

where $J$ is the total angular momentum of $A$. The superscript $C$ indicates the presence of the Coulomb interaction between the fragments; $W_{-\kappa, l+1 / 2}$ is a Whittaker function, which when divided by $\rho$ is normalized to unity by $N_{W}$ for $l=0$ after $\mathrm{d}^{3} \rho$ integration. $\mathrm{Y}_{m}^{[l]}(\hat{\rho})=(-i)^{l} \mathrm{Y}_{l m}(\hat{\rho}) ;$ while $\varphi$ and $\psi$ represent the internal wave functions of $\mathrm{x}$ and $\mathrm{y}$. The Coulomb parameter

$$
\kappa=\frac{\mathrm{Z}_{x} \mathrm{Z}_{y} \mu_{x y} \alpha}{\mu^{C}}
$$

where $\alpha$ is the fine structure constant and the binding energy of $\mathrm{x}$ and $\mathrm{y}$ within $\mathrm{A}$ is given by $\mathrm{B}=\left(\mu^{C}\right)^{2} / 2 \mu_{x y^{*}} \cdot$ The reduced mass of the $\mathrm{x}-\mathrm{y}$ system is $\mu_{x y^{\circ}}$. We have purposedly limited $l$ to the values 0 and 2 , i.e., S- and D-wave asymptotic normalization constants, because the $A=3-6$ nuclei all have positive parity and $J=0,1 / 2$, or 1 , thus limiting $l$ to 0 or 2 . If one of the fragments is not charged, then $C_{l}^{C} \rightarrow C_{l}, \mu^{C} \rightarrow \mu$, and

$$
i^{l} N_{W} \frac{W-\kappa, l+1 / 2\left(2 \mu^{C} \rho\right)}{\rho} \Rightarrow-\sqrt{\frac{\mu^{3}}{2 \pi}} h_{l}^{(1)}(i \mu \rho) \text {, }
$$

where $h_{l}^{(1)}(i \mu \rho)$ is a spherical Hankel function of the first kind.

Physically, an asymptotic normalization constant is a dimensionless quantity that echoes the internal dynamics present in the wave function through overall normalization. Asymptotic normalization constants are defined such that the S-wave asymptotic norm is unity when the effective nuclear interaction in the asymptotic channel of interest is an S-wave zero-range interaction independent of the presence of the Coulomb interaction. The scale is set by the normalized, S-wave, zero-range comparison functions, i.e., the quantities in Eq. (3) with $l=0$. Since the zero-range limit is never achieved, owing to the boundary condition on the wave function at the origin, asymptotic normalization constants differ from unity. Though the individual $l$-wave asymptotic normalization constants have intrinsic interest, the ratio of the D-wave to S-wave asymptotic normalization constants is the predominant quantity used to quantify the D-state presence.

Closely allied with the asymptotic normalization constants are the DWBA parameters $D_{l} / 12 /$. In transfer or pickup reactions, e.g., $(\mathrm{d}, \alpha)$, analyzed as a single-step process in DWBA, the key quantity that enters with respect to the projectile vertex is the overlap

$$
\begin{aligned}
& <\rho \varphi_{m_{x}}^{\left[j_{x}\right]} \psi_{m_{y}}^{\left[j_{y}\right]}\left|\Psi_{M}^{[J]}\right\rangle=\underset{\substack{l=0,2 \\
m_{l}}}{\sum_{\boldsymbol{m}}^{J}} i^{l} U_{l}(\rho)<j_{x} m_{x} j_{y} m_{y} \mid J m> \\
& \times<l m_{l} J m \mid J m>\sqrt{4 \pi} \mathrm{Y}_{m_{l}}^{[l]}(\hat{\rho}),
\end{aligned}
$$

where $U_{l}(\rho)$ is the $l$-th wave effective $\mathrm{x}-\mathrm{y}$ wave function in $\mathrm{A}$ and

$$
\lim _{\rho \rightarrow \infty} U_{l}(\rho) \Rightarrow C_{l}^{C} N_{W} \frac{W_{-\kappa, l+1 / 2}\left(2 \mu^{C} \rho\right)}{\rho} \equiv_{\kappa} C_{l} i^{l+2} \sqrt{\frac{\mu^{3}}{2 \pi}} h_{l}^{(1)}(i \mu \rho)
$$

The $\mathrm{A} \rightarrow \mathrm{X}+\mathrm{y}$ momentum distribution amplitude is obtained from the Fourier transform of the overlap in Eq. (4). The momentum distribution amplitude has exactly the same form as in Eq. (4) except that $\rho$ on the left-hand side is replace by $q$, where $q$ is the relative momentum of the $x-y$ pair in $A$, and on the right-hand side, $\rho$ is replaced by $q$, the $i^{l}$ is not present, and $U_{l}(\rho)$ is replaced by the partial-wave momentum distribution amplitude $f_{l}(q)$ defined as follows:

$$
f_{l}(\mathrm{q}) \equiv \sqrt{\frac{2}{\pi}} \int_{0}^{\infty} \rho^{2} \mathrm{~d} \rho j_{l}(\mathrm{q} \rho) U_{l}(\rho)
$$

In terms of the $f_{l}(q)$, with or without the Coulomb interaction present between $\mathrm{x}$ and $\mathrm{y}$,

$$
C_{l}=i^{i} 2 \pi i \mu^{1 / 2} \lim _{q \rightarrow i \mu}(q-i \mu) f_{l}(q)
$$




$$
\begin{gathered}
\eta=\frac{C_{2}}{C_{0}}, \\
D_{0}=\sqrt{8 \pi^{3}} . \mathrm{B} f_{0}(0)=\mathrm{B} 4 \pi \int_{0}^{\infty} \rho^{2} \mathrm{~d} \rho U_{0}(\rho),
\end{gathered}
$$

and

$$
D_{2}=\lim _{q \rightarrow 0}\left\{\frac{f_{2}(q)}{q^{2} f_{0}(q)}\right\}=\frac{1}{15} \frac{\int_{0}^{\infty} \rho^{4} \mathrm{~d} \rho U_{2}(\rho)}{\int_{0}^{\infty} \rho^{2} \mathrm{~d} \rho U_{0}(\rho)}
$$

(Simply add a superscript $\mathrm{C}$ to $C_{l}, \mu, D_{0}$, and $D_{2}$ when both $\mathrm{x}$ and $\mathrm{y}$ are charged.) In equation (9), we adopt the definition for $D_{2}$ originally adopted by Johnson and Santos $/ 12 \%$. Within this convention, $\eta$ and $D_{2}$ have the same sign. Unlike the DWBA parameters, which can be extracted from the momentum distribution amplitudes at $q=0$, the $C_{l}$ and $\eta$ require analytic continuation of $q$ to the bound-state pole of $f_{l}(q)$. However, to the extent that the asymptotic forms of Eq. (5) can be used to determine the $f_{l}(q)$ in ratio,

$$
D_{2}^{C} \approx \frac{\eta^{C}}{\left(\mu^{C}\right)^{2}} \boldsymbol{F}(\kappa) \equiv \widetilde{D}_{2}^{C}{ }_{\kappa} \equiv_{0} \widetilde{D}_{2}=\frac{\eta}{\mu^{2}},
$$

where $F(\kappa)$ is a ratio of hypergeometric functions /13/. The applicability of Eqs. (11) must be checked for each case. For the deuteron, Eq. (11) holds to $1 \%$ or better $/ 14,9 /$.

\section{3 - 's-SHELL' NUCLEI}

${ }^{2} \mathrm{H}$ :

\section{Theory}

Two observables associated with the structure of the deuteron give clear evidence of the tensor interaction between two nucleons and of the existence of a $D$-state component in the deuteron wave function: $Q_{d}$, the deuteron quadrupole moment, and $\eta_{d}$ (or $\mathrm{D}_{2}$ ). Amado $/ 3 /$ and Friar $/ 4 /$ have pointed out that, unlike $\mathrm{Q}_{d}$ and $\eta_{d}$, the percentage $\mathrm{D}$-state, $\mathrm{P}_{D}\left({ }^{2} \mathrm{H}\right)$, in the deuteron wave function is not an observable. The nonzero values of $\mathrm{Q}_{d}$ and $\eta_{d}$ assure that $\mathrm{P}_{D}\left({ }^{2} \mathrm{H}\right)$ is nonzero, but neither constrains its value. Both $Q_{d}$ and $\eta_{d}$ depend primarily on the part of the deuteron wave function that is external to the range of the nuclear interaction. $P_{D}\left({ }^{2} \mathrm{H}\right)$, being a normalization integral, depends on the whole wave function -- interior and exterior parts. By investigating the singularities of amplitudes, Amado shows that determination of the interior part of a wave function requires a dynamical model. Within a given model, $\mathrm{P}_{D}\left({ }^{2} \mathrm{H}\right)$ has a fixed value, but a different dynamical model fitted to the same empirical data will generally yield a different value of $\mathrm{P}_{D}\left({ }^{2} \mathrm{H}\right)$. Equivalently, Friar used the fact that unitary transformations applied to operators and wave functions leave observables unaltered. By that approach, he showed that $\mathrm{P}_{D}\left({ }^{2} \mathrm{H}\right)$ depends on the unitary transformation. Nevertheless, $\mathrm{P}_{D}\left({ }^{2} \mathrm{H}\right)$ can serve as a parameter that characterizes a particular two-nucleon interaction.

By now, the nature of $\mathrm{Q}_{d}$ and $\eta_{d}$ is well explicated $/ 5 / . \mathrm{Q}_{d}$ and $\eta_{d}$ can be understood in terms of the two-nucleon tensor interaction, specifically, the OPE piece of the tensor interaction, to an accuracy of a few percent. By means of the Schwartz inequality, it is possible to exploit the tight theoretical association between $\eta_{d}$ and the OPE potential to place rather narrow bounds on the value of $\eta_{d}$, specifically, $0.0268 \pm 0.0007 / 15 /$. Moreover, by means of an exact integral relation that relates $\eta_{d}$ directly to the two-nucleon tensor interaction and the S-wave component of the deuteron wave function, Ericson and Rosa-Clot /5/ have shown that, with consideration of corrections to the OPE approximation to the tensor interaction and point nucleons, $\eta_{d}=0.0259 \pm 0.0003$. The error on this latter value was indicated as coming mainly from the value for the $\pi \mathrm{N}$ coupling constant, but the size of this quoted error has been questioned /15-18/. Looking at $2 \pi$ corrections to the OPE approximation, Ericson and Rosa-Clot find that the OPE result is lowered by $\sim 4 \%$. In the Ericson/Rosa-Clot analysis, the binding energy of ${ }^{2} \mathrm{H}$ is kept fixed while the sensitivity of $\eta_{d}$ to changes of parameters in the interaction and the S-wave component of the wave function was estimated. One learns that there is a strong dependence of $\eta_{d}$ on the $\pi \mathrm{N}$ coupling constant. In fact, for that reason, one must be careful in discussing the values of $\eta_{d}$ from potential models to assure that the $\pi \mathrm{N}$ coupling constant is the same in all cases. Based on the various local, energy-independent potential models of the twonucleon interaction, consistent with the experimental effective range and OPE, Ericson and Rosa-Clot propose that there is a linear relationship between $\overline{\mathrm{Q}}_{d} / C_{0}^{2}$ and $\bar{\eta}_{d}$ that can be tested with a higher precision determination of $\eta_{d}$. (The bar over $\mathrm{Q}_{d}$ and $\eta_{d}$ is to indicate that each has been scaled by $0.078 / \mathrm{f}^{2}$ to account for the different values of the $\pi \mathrm{N}$ coupling constant used [most current value $/ 19 /$ is $\mathrm{f}^{2}=0.0776 \pm 0.0009$ ]). Heuristically, one can get a hint at such a relationship by substituting the asymptotic forms for the deuteron wave function components (See Eq. (5) above) into the equation for the deuteron quadrupole moment. Moreover, this relationship indicates clearly that the sign of $\eta_{d}$ is directly related to the sign of $\mathrm{Q}_{d}=0.2859 \pm 0.0003 \mathrm{fm}^{2} / 7 /$ and suggests the value $\eta_{d}=0.0264 \pm 0.003 / 5 /$.

As we indicated above, the Ericson and Rosa-Clot result assumes point particles, that is, there are no $\pi \mathrm{N}$ form factors to account for the finite size of the nucleons. To estimate the effect of this approximation, they terminate the OPE potential at some minimum distance where the nucleons are supposed to lose their identity, i.e., a range at which form factors would be expected to introduce modifications. Their result is that the value of $\eta_{d}$ decreases by an amount which depends on the cutoff radius employed. For example, a cutoff radius of $0.8 \mathrm{fm}$ would give the value $\eta_{d}=0.0242 / 15,20 /$. Certainly, this finding is 
a surprise, since effects of form factors are usually thought to be confined to small nucleon separation distances, and thus would have no or very limited effect on external observables. This motivated Ballot et al. $/ 6 /$ to study this issue further.

Specifically, Ballot et al. construct a potential containing an OPE tail that is regularized at the origin by means of three parameters that allow them to construct several families of deuteron wave functions. With these wave functions, an assessment is made of the influence of the inner parts of the potential on the root-mean-square radius $\left(\mathrm{r}_{m}\right), \mathrm{Q}_{d}$, and $\eta_{d}$. This is done by keeping the $\pi \mathrm{N}$ coupling constant, the pion mass, and the ${ }^{2} \mathrm{H}$ binding energy fixed. Since the ${ }^{2} \mathrm{H}$ binding energy is determined by the interaction, the three parameters of the potential are related to each other. The three parameters are the two cutoff parameters of the central and tensor components of the interaction and another parameter, $\delta$, multiplying the contribution to the central potential that becomes the OPE contact interaction when the central cutoff parameter approaches $\infty$. The cutoffs are introduced by simply multiplying the $\pi \mathrm{N}$ coupling constant by an 'effective' monopole form factor. So, as their cutoffs approach $\infty$, they recover the standard OPE potential. Within this framework, they find that the ratio $r_{m} / C_{0}$ is remarkably stable under changes in the inner parts of the potential, i.e., there is a very strong linear correlation between these two quantities. This has been noted previously and has been used to determine $C_{0}$ from $r_{m} / 21,17 /$. For $\eta_{d}$, there is a dependence on the inner parts of the potential at the few percent level. At the worst, it is $\leq 5 \%$. In fact, if the central potential cutoff value is maintained within a more conservative range, the variation induced in $\eta_{d}$ is considerably reduced. This agrees qualitatively with the results of Friar et al. where no distinction is made between the cutoff parameters between the central and tensor components of the interaction $/ 22 /$. The results for $\mathrm{Q}_{d} / C_{0}^{2}$ are similar. How does the latter ratio and $\eta_{d}$ actually correlate? Once the central cutoff parameter is $\geq 1200 \mathrm{MeV}$, all curves that vary with $\delta$ merge into a single straight line. This line is universal. Since it is independent of the central cutoff parameter and $\delta$, the relationship of the the three potential parameters means it is independent of the tensor cutoff parameter as well. The universal line derives irom the joint action of the tails of both central and tensor potentials. How does $\eta_{d}$ depend on the tensor cutoff parameter? There is a universal curve when the central cutoff parameter is not too small, i.e., the central potential already possesses the OPE potential tail. Thus, the curve depends on the OPE potential tail, but not its internal structure. However, the fact that it is a curve indicates a clear dependence of $\underline{\eta}_{d}$ on the non-OPE potential structure of the tensor potential. Therefore, in principle, the tensor cutoff parameter can be constrained by an experimental value for $\eta_{d}$.

On a more speculative level, it has been shown by Guichon and Miller /23/ that the reduction of the deuteron D-state by loss of pion exchange within the cutoff radius can be compensated partially by tensor interactions generated by constituent quarks and gluons. Within the context of a properly antisymmetrized 6-quark bag model, they have been able to show that larger cutoff radii are not ruled out, i.e., $\eta_{d}$ does not get as drastically reduced as in the case of the Ericson/Rosa-Clot analysis /5/. In the Guichon-Miller model, a cutoff radius of $0.8 \mathrm{fm}$ leads to a reduction in $\eta_{d}$ of only about $1 \%$ compared to the case of point nucleons cited above.

Finally, one must remember that it is possible that other components in the deuteron wave function play a role in the prediction of the value for $\eta_{d}$. For example, it is possible to have $\Delta \Delta$ or $N^{*}$ components in the deuteron wave function. Phenomenological models of the nucleon-nucleon (NN) interaction that include these latter channels lead to deuteron wave functions that generate values for $\eta_{d}$ that are somewhat smaller than those obtained from standard NN interactions $/ 24,25 /$. Such models that fit the usual static properties of the deuteron lead to values for $\eta_{d}$ in the range 0.0251 to 0.0258 . Clearly, the role of $\Delta$ and $N^{*}$ components in the deuteron wave function, or equivalently, the whole question of the role of nucleon internal degrees of freedom in calculating the observables associated with the deuteron, requires further and thorough study.

\section{Experiment}

Three new values of $\eta_{d}$ have recently been extracted from experiment; each by a different method. The three methods of extraction involve DWBA analysis of subCoulomb $(\vec{d}, \mathrm{p})$ reactions, pole extrapolation of ${ }^{2} \mathrm{H}(\mathrm{d}, \mathrm{p})^{3} \mathrm{H}$ polarization observables, and extraction of the residue at the deuteron pole from a low-energy phase-shift analysis of neutron-proton scattering, respectively. The three values extracted for $\eta_{d}$ are as follows:
(a) $0.0256 \pm 0.0004$
(b) $0.0273 \pm 0.0005$
$(\vec{d}, \mathrm{p})$
$/ 26 /$,
(c) $0.02712 \pm 0.00022$
${ }^{2} \mathrm{H}(\vec{d}, \mathrm{p}){ }^{3} \mathrm{H}$
np phase-shift analysis
$128 /$.

Clearly, since the quoted errors imply a high precision in the determination of these values, a conflict clearly exists between (a) and (b) or (c), while (b) and (c) appear to be compatible with each other. The ultimate resolution of these discrepancies will require further work. However, we review briefly what underlies the extraction of each value.

Value (a) from Rodning and Knutson /26/ is extracted by means of the standard DWBA analysis of subCoulomb (d,p) transfer reaction polarization observables at different bombarding energies, from two different targets, and to two different final states for each target. The new value is not fully consistent with the earlier value $(0.0271 \pm 0.0008)$ obtained by Knutson and his collaborators $/ 29 /$. So why should we believe the new value? The authors argue that they have a partial understanding of the difference in that they have recalibrated their polarimeter, which leads to a $1.2 \%$ change in the normalization of $\mathrm{T}_{20}$ at the common energy of $7 \mathrm{MeV}$, and they require the tensor interaction in the distorting potential for the deuteron to be consistent with the elastic scattering measurements, which leads to a $0.6 \%$ change towards the new value. The DWBA calculation is full finite-range with the above tensor interaction, a long-range tensor potential to account for the interaction of the deuteron quadrupole moment with the electric-field gradient of the target nucleus, and a long-range potential that accounts for the polarization of the deuteron in the Coulomb field of the target nucleus with appropriate modification of the deuteron wave function. Since the incident energies are in the subCoulomb regime, the distortion mainly is from the Coulomb interaction. Within this framework, and with the Reid Soft Core (RSC) /30/ wave function serving as the deuteron wave function $\left(\eta_{d}=0.0263\right)$, ten statistically independent values of $\eta_{d}$ are extracted. A weighted average of the 
values extracted from each of the ten angular distributions leads to the above result. Considerable effort is put into assessing the systematic errors. However, it would be interesting to know the sensitivity of the result to the use of the RSC wave function as opposed to the Paris /31/ or Bonn /32/ wave functions.

The second value quoted above is extracted from work by Borbély et al. /27/ where they give a detailed justification for the analytic continuation of empirical polarization observables and respond to their critics. They acknowledge that the criticism of the analytic continuation of the observables in ${ }^{1} \mathrm{H}(\mathrm{d}, \mathrm{p})^{2} \mathrm{H}$ is justified and that this particular case requires further work. However, they argue that similar criticism /33/ carried over to the polarization observables in ${ }^{2} \mathrm{H}(\mathrm{d}, \mathrm{p})^{3} \mathrm{H}$ is not justified. Specifically, they address the issues of 1.) Incorrect handling of the Coulomb renormalization at the vertices; 2.) Large truncation errors in extrapolating the fitting function; and 3.) Unreliable results caused by the cut (Coulomb) beginning at the nucleon-exchange singularity. The key aspect of their approach is that the cross section for the above reaction is dominated by the two possible exchange peaks: neutron exchange or proton exchange. Then, by looking at the pole diagrams that describe these exchanges, they argue that the tensor analyzing powers (TAP's) are independent of the Coulomb renormalization factor(s), because the Coulomb renormalization factors are independent of the vertex orbital momenta (in lowest order) $/ 34,35 /$, thus they cancel in the TAP's. On the other hand, truncation errors would imply background singularities coming from other Feynman diagrams, e.g., instead of an exchange diagram, a pickup process leading to the same final states. However, they argue that the kinematic factor for the pickup process is very small in the TAP's compared to stripping, thus stripping totally dominates. Nevertheless, they check this dominance with different expansion methods, with and without conformal mapping, and by the use of suppression factors. They conclude that conditions are favorable for the use of the method and that the truncation error is at most $\sim 1 \%$. Finally, the branch point character of the exchange singularity which has its source in the Coulomb interaction is more serious. In the cross section, the main second order pole coming from the exchange process is still present, but the interference of the exchange amplitude with any background amplitude leads to an interference term that has a branch point rather than a simple pole. Therefore, in a naive extrapolation point of view, analytic continuation of $\left(z-z_{p}\right)^{2} \sigma(z)$ is not possible since the singularity in the interference term is incompletely removed. However, by using their alternative method called the 'asymptotic coefficients method', they argue that they did not find any trace of the branch-point contribution within statistical errors. Then, after consideration of other possible systematic errors, they give the value listed above with what they consider a conservative estimate of the error.

Finally, Stoks, et al. /28/ carry out an energy-dependent phase-shift analysis of all low-energy np scattering data below $\mathrm{T}_{l a b}=30 \mathrm{MeV}$. With their fit, the S-matrix elements of the coupled ${ }^{3} S_{1}{ }^{3} D_{1}$ channels are determined which, in turn, allows calculation of the residue at the deuteron pole and extraction of $\eta_{d}$. By this method the third value given above is obtained. It should be mentioned that the errors treated in the phase-shift analysis are purely statistical. A thorough investigation of any systematic errors is required for a reliable comparison with values (a) and (b).

${ }^{3} \mathrm{H} /{ }^{3} \mathrm{He}$ :

Theory

In contrast to the deuteron, ${ }^{3} \mathrm{H}$ and ${ }^{3} \mathrm{He}$ possess no quadrupole moment, and as a consequence display directly a D-state component in their respective wave functions only through nonzero ratios of $\mathrm{D}$-wave to $\mathrm{S}$-wave asymptotic normalization constants, hereafter called $\eta_{t}$ and $\eta_{t}^{C}$, respectively. In addition, there are the two DWBA parameters $D_{2}^{t}$ and $D_{2}^{t C}$.

Reliable theoretical predictions of $\eta_{t}$ and $\eta_{t}^{C}\left(D_{2}^{t}\right.$ and $\left.D_{2}^{t} C\right)$ for the trinucleons have progressed significantly over the last ten years, as can be seen in Table 1 below. The objective is to use the best phenomenological two-nucleon interactions that possess the one-pion-exchange contribution, sometimes supplemented with a three-nucleon interaction, to obtain as complete (or as converged) a three-nucleon wave function as possible. This has been demonstrated to be achieved when all two-body partial waves up to $\mathrm{j} \leq 4$ (34 three-body channels) have been included. Then the binding energy is converged to within 10 $\mathrm{keV} / 36 /$. Such wave functions are then used in integral relations $/ 37,38,13 /$ to determine the asymptotic normalization constants.

The most recent theoretical values for $\eta / 39-42 /$ are significantly larger than the early 5-channel Reid-Soft Core results $/ 13,43,44 /$, because they are extracted by either 'scaling' the results of many models to the experimental binding energy /4042 / or from a 34-channel calculation where the binding energy is not far from the experimental value /40/. Ishikawa and Sasakawa $/ 41,42 /$ were the first to show that $\eta_{t}$ is linearly correlated with the ${ }^{3} \mathrm{H}$. binding energy without regard to the underlying two-nucleon interaction. Their 'data set' includes 5-, 18-, 26-, and 34-channel calculations, with and without a $3 \mathrm{~N}$ interaction. Then, by fitting this theoretical data linearly, they obtain $\eta_{t}=-0.0432 \pm 0.0015$ at $8.48 \mathrm{MeV}$. Friar et al. /39/ did a similar analysis for both ${ }^{3} \mathrm{H}$ and ${ }^{3} \mathrm{He}$; their value for $\eta_{t}$ is $\sim 7 \%$ larger in magnitude than that of Ishikawa and Sasakawa /41-42/. The $\eta_{t}$ result from the Bonn (1987) potential (34 channels), where the ${ }^{3} \mathrm{H}$ binding energy is $8.34 \mathrm{MeV}$, is consistent with the work of Friar et al.

The idea underlying the linear fit of $\eta_{t}$ as a function of the ${ }^{3} \mathrm{H}$ binding energy is that, if $\eta_{t}$ (or $\eta_{t}^{C}$ ) scales with energy, then fits to these theoretical 'data sets', evaluated at the experimental binding energy, should provide the best theoretical estimate of $\eta_{t}$ or $\eta_{t}^{C}$. Of course, that assumes the correctness of the physies in the models. For example, on the basis of an apparent linear correlation of $\eta_{t}$ and $\eta_{d}$, as originally suggested by Ericson and Rosa-Clot $/ 5 /$, Frederico et al. $/ 50 /$ argue that since $\eta_{t}$ is sensitive to $\eta_{d}$, it is not sufficient to specify the ${ }^{3} \mathrm{H}$ binding energy to determine $\eta_{t}$ uniquely. Specifically, they extract on the basis of a wide class of models, with both local and nonlocal separable potentials, $\eta_{t} / \eta_{d}=-1.68 \pm 0.04$ at the triton binding energy values. Their point is especially important in light of the uncertainties in the empirical determination of $\eta_{d}$ discussed above. Nevertheless, the calculations by Ishikawa/Sasakawa /41-42/ and Friar et al. /39/ have a very small dispersion with respect to a linear fit of $\eta_{t}$ vs. binding energy, because only a small band of $\eta_{d}$ values is involved in the NN potentials considered $\left(\eta_{d}=0.0263 \pm 0.0003\right)$. More recently, Adhikari and Frederico /51/ have extended their analysis to 
include $D_{2}^{t}$ and find that $D_{2}^{t} / \eta_{d}=-7.9 \pm 0.4 \mathrm{fm}^{2}$. From this new result, combined with their earlier result for $\eta_{t}$, they check the approximation given in Eq. (11). They find that $D_{2}^{t} \mu^{2} / \eta_{t}=0.95 \pm 0.06$, i.e., to within one standard deviation, Eq. (11) is probably better than a $5 \%$ approximation in the case of ${ }^{3} \mathrm{H}$.

Table 1 Theoretical values of ${ }^{3} \mathrm{H}$ and ${ }^{3} \mathrm{He}$ D-state observables

\begin{tabular}{|c|c|c|c|c|}
\hline Quantity & ${ }^{3} \mathrm{H}$ & ${ }^{3} \mathrm{He}$ & Method & Reference \\
\hline \multirow[t]{2}{*}{$\eta$} & $\begin{array}{c}-0.046 \pm 0.001 \\
-0.043\end{array}$ & $-0.043 \pm 0.001$ & $\begin{array}{l}\mathrm{BF} / \mathrm{EB}^{a} \\
\text { Bonn NN }(34)^{b} \\
\quad(\mathrm{OBEPQ})\end{array}$ & $\begin{array}{l}/ 39 / \\
/ 40 /\end{array}$ \\
\hline & $\begin{array}{c}-0.0432 \pm 0.0015 \\
-0.0374 \\
-0.037 \\
-0.037 \pm 0.002\end{array}$ & $\begin{array}{c}-0.0344 \\
--- \\
----\end{array}$ & $\begin{array}{l}\text { BF/EB } \\
\text { RSC5 }^{\circ} \\
\text { RSC5 } \\
\text { RSC5 }\end{array}$ & $\begin{array}{l}\mid 41-42 / \\
/ 13 / \\
/ 43 / \\
/ 44 /\end{array}$ \\
\hline$D_{2}\left(\mathrm{fm}^{2}\right)$ & $\begin{array}{c}-0.20 \pm 0.02 \\
-0.23 \\
-0.27 \\
-0.21\end{array}$ & ב-ב & $\begin{array}{l}\mathrm{BF} / \mathrm{EB}^{d} \\
\mathrm{AV}(\mathrm{UR})^{e} \\
\mathrm{UR}^{\mathrm{U}(\mathrm{UR})^{f}} \\
\mathrm{RSCV}_{\mathbf{8}}, \mathrm{AV}_{\mathbf{1 4}}, \mathrm{TRS}^{g}\end{array}$ & $\begin{array}{l}\mid 41-42 / \\
/ 45 / \\
/ 45 / \\
/ 48 /\end{array}$ \\
\hline$\widetilde{D}_{2}\left(\mathrm{fm}^{2}\right)$ & $\begin{aligned}-0.229 & \pm 0.005 \\
-0.22 & \pm 0.01\end{aligned}$ & $-0.238 \pm 0.005$ & $\begin{array}{l}\mathrm{BF} / \mathrm{EB} \\
\mathrm{BF} / \mathrm{EB}^{d}\end{array}$ & $\begin{array}{l}/ 39 / \\
\mid 41-42 /\end{array}$ \\
\hline
\end{tabular}

\footnotetext{
${ }^{a}$ Best fit/Experimental binding energy based on scaling of models with energy.

${ }^{b}$ Bonn NN potential, 34 channels, momentum space $/ 32 /$.

${ }^{c}$ Reid-Soft-Core NN potential, 5 channels $/ 30 /$.

${ }^{d}$ Based on least squares fit to tabulated values.

eArgonne NN interaction with phenomenological $3 \mathrm{~N}$ interaction /46/.

${ }^{f}$ Urbana NN interaction with phenomenological $3 \mathrm{~N}$ interaction $/ 47 /$.

${ }^{g}$ TRS $/ 49 /$.
}

One aspect of the theoretical results for ${ }^{3} \mathrm{H}$ and ${ }^{3} \mathrm{He}$ should be brought out at this time: signs. As we mentioned in the section on formalism, our $\eta$ 's and $D_{2}$ 's have the same sign. Above, you will note that the values of these two quantities for the 3-body nuclei are of opposite sign to that of ${ }^{2} \mathrm{H}$. For the three-body nuclei, the sign is dependent on the coupling order that one chooses in Eq. (4). Negative signs for $\eta$ and $D_{2}$ correspond to taking the deuteron as particle $\mathrm{x}$ and the nucleon as particle y. If we simply reverse that convention, we shall end up with $\eta$ and $D_{2}$ being positive. In fact, it is this latter convention that has been used in the three-body papers referenced in Table 1 . Nevertheless, a case based on empirical evidence can be made for choosing the convention that leads to the negative values. However, it must be emphasized that once the coupling convention is specified, the three-body dynamics ultimately determines the sign. In other words, the theoretical results not only predict the magnitudes, but also the signs of the results, given a coupling convention. For the nuclei under discussion in this paper, it is only the three-body nuclei where this coupling order is an issue. For all the other cases, since the overall angular momentum is an integer, the coupling order does not enter.

\section{Experiment}

Considerable effort has been extended since the early 1970 's in an attempt to extract from measurements information about the $\mathrm{D}$-wave component of the ${ }^{3} \mathrm{H} \rightarrow \mathrm{n}+\mathrm{d}$ and ${ }^{3} \mathrm{He} \rightarrow \mathrm{p}+\mathrm{d}$ overlaps, or equivalently, vertices. Values for $\eta_{t}$ and $\eta_{i}^{C}$, as well as $D_{2}^{t}$ and $D_{2}^{t} C$, are available. I list some of the most current values in Table 2 below.

The first evidence for the presence and significance of the D-state component in the ${ }^{3} \mathrm{H} \rightarrow \mathrm{n}+\mathrm{d}$ overlap was provided by Knutson et al. $/ 52 /$ by means of $(\mathrm{d}, \mathrm{t})$ transfer reactions with polarized deuterons. It was demonstrated that the TAP's are as observed primarily because of the D-state term in the ${ }^{3} \mathrm{H} \rightarrow \mathrm{n}+\mathrm{d}$ overlap. This conclusion was reached by means of DWBA calculations, where the local energy approximation (LEA) is used to account approximately for finite-range effects at the ${ }^{3} \mathrm{H} \rightarrow \mathrm{n}+\mathrm{d}$ vertex. The vertex amplitude is obtained by multiplying the momentum-space equivalent of Eq. (4) by $-\left(q^{2} / 2 \mu_{n d}+B\right)$. The LEA approximation is then obtained by expanding about $q=0$ which is facilitated by Eq. (6) and the definitions in Eqs. (9) and (10). What one finds is that the D-state effect depends only on $D_{2}$. For $D_{2} \equiv 0$, the DWBA calculations greatly underestimate the TAP's. Though uncertainties are present in extracting a value for $D_{2}^{t}$, the 'best fit' value turned out to be $-0.24 \mathrm{fm}^{2}$.

Following the first extraction of $D_{2}^{t}$, measurements and analysis focused on whether $D_{2}^{t}$ differed significantly from $D_{2}^{t} C$, whether subCoulomb energies for the incident projectile led to more reliable results, what role the tensor component played in the distorting potential for the incident deuteron, and how important exact finite-range calculations are compared to the LEA. The interested reader can find a complete list of references and a complete listing of results in Table 1 of Ref. /10/. Initially, it appeared that the ${ }^{3} \mathrm{H}$ and ${ }^{3} \mathrm{He} D_{2}$ values might differ significantly from each other, but with further measurements and refined analyses no differences are observed within errors that are typically $\geq 20 \%$ (see Table 2 above). Arguments have been advanced for making measurements at subCoulomb energies /59/ because the DWBA analysis should 
be most reliable (reactions are peripheral), and the weaker distortion effects should minimize spin-dependent force contributions for the incident deuteron. Though the results appear to be stable (see Ref. /10/) at subCoulomb energies, the analyses are limited to LEA without $T_{R}$. Introducing $T_{R}$ into the DWBA analysis indicates that the TAP's do not change significantly in shape $/ 60 /$, but their magnitude increases and thus reduces the magnitude of $D_{2}$ needed for the 'best fit' to the data. The LEA approximation seems to be valid at forward angles and is more likely to break down toward backward angles for the TAP's, where full finite-range effects should play a role $/ 61 /$.

Table 2 Experimental values of ${ }^{3} \mathrm{H}$ and ${ }^{3} \mathrm{He}$ D-state observables

\begin{tabular}{|c|c|c|c|c|}
\hline Quantity & ${ }^{3} \mathrm{H}$ & ${ }^{3} \mathrm{He}$ & Method & Reference \\
\hline \multirow[t]{5}{*}{$\eta$} & $-0.050 \pm 0.006$ & $-0.035 \pm 0.006$ & $\begin{array}{l}\mathrm{AC} \text { of } \mathrm{TAP}^{\alpha} \\
{ }^{4} \mathrm{He}\left(\mathrm{d},{ }^{3} \mathrm{He}\right)^{3} \mathrm{H}\end{array}$ & $/ 53 /$ \\
\hline & $-0.051 \pm 0.005$ & ---_- & $\begin{array}{l}\text { AC of TAP } \\
{ }^{2} \mathrm{H}(\mathrm{d}, \mathrm{p})^{3} \mathrm{H}\end{array}$ & $/ 54 /$ \\
\hline & $-0.048 \pm 0.007$ & ------ & $\begin{array}{l}\text { AC of TAP } \\
{ }^{2} \mathrm{H}(\mathrm{d}, \mathrm{p})^{3} \mathrm{H}\end{array}$ & $/ 55 /$ \\
\hline & ------- & $-0.048 \pm 0.006$ & $\begin{array}{l}\mathrm{EFR} / \mathrm{DWBA} \mathrm{A}^{b} \\
{ }^{32} \mathrm{~S}\left(\mathrm{~d},{ }^{3} \mathrm{He}\right)^{31} \mathrm{P}\end{array}$ & $/ 56 /$ \\
\hline & $-0.050 \pm 0.010$ & $-0.042 \pm 0.007$ & $\begin{array}{l}\text { EFR/DWBA }{ }^{b} \\
{ }^{31} \mathrm{P}\left(\mathrm{d},{ }^{3} \mathrm{H}\right) \\
{ }^{31} \mathrm{P}\left(\mathrm{d},{ }^{3} \mathrm{He}\right)\end{array}$ & $/ 57 /$ \\
\hline \multirow[t]{2}{*}{$D_{2}\left(\mathrm{fm}^{2}\right)$} & $-0.25 \pm 0.05$ & $-0.24 \pm 0.04$ & $\begin{array}{l}\text { EFR/DWBA } \\
{ }^{31} \mathrm{P}\left(\mathrm{d},{ }^{3} \mathrm{H}\right) \\
{ }^{31} \mathrm{P}\left(\mathrm{d},{ }^{3} \mathrm{He}\right)\end{array}$ & $/ 57 /$ \\
\hline & $-0.20 \pm 0.04$ & $-0.25 \pm 0.05$ & \multicolumn{2}{|c|}{$\begin{array}{l}\text { EFR/ DWBA with } \mathrm{T}_{R}^{c} / 58 / \\
{ }^{40} \mathrm{Ca}\left(\mathrm{d},{ }^{3} \mathrm{H}\right) \\
{ }^{40} \mathrm{Ca}\left(\mathrm{d},{ }^{3} \mathrm{He}\right)\end{array}$} \\
\hline
\end{tabular}

\footnotetext{
Analytic continuation of tensor analyzing powers.

${ }^{b}$ Exact Finite Range/Distorted-Wave Born Approximation. The $\eta$ values are determined from model wave functions for the three-body vertices.

${ }^{c}$ With tensor component in the deuteron distorting potential.
}

Evidently, there are a number of uncertainties in the extraction of $D_{2}^{t}$ and $D_{2}^{t C}$ from transfer reactions. Prominent among these is how well defined are the distorting potentials for the deuteron and three-nucleon nuclei. This includes the question of the $T_{R}$. There remains the issue of whether Coulomb polarization effects on the incident deuteron are significant as well. We noted above that in the extraction of the deuteron $\eta_{d}$ these questions concerned Rodning and Knutson. Until these matters are more completely understood, we must settle for a determination of $D_{2}$ uncertain by at least $20 \%$. In fact, the error may be greater than this owing to the unknown systematic errors that arise in the limitations in completely describing the tensor analyzing powers with existing theory. (To a more limited extent, this may also apply to the extraction of $\eta_{d}$ by this method.) Nevertheless, within the coupling scheme adopted, the signs of $D_{2}^{t}$ and $D_{2}^{t} C^{c}$ have been determined to be negative.

In contrast, $\eta_{t}$ appears to be more precisely determined, i.e., to within $\sim 12 \% / 53-55 /$, as can be seen in Table 2 . In addition, we can see that, within the constraints of using model wave functions to handle an extraction of $\eta_{t}$ from the transfer reaction results $/ 57 /$, the AC of TAP's results are consistent with the latest transfer reaction results. However, $\eta_{t}^{C}$ is reported to be $30 \%$ smaller than $\eta_{t}$ for the AC of TAP's results, whereas the transfer reaction analyses indicate that $\eta_{t}$ is comparable to $\eta_{t}$. Of course, one strength of the AC of TAP's results is that $\eta_{t}$ and $\eta_{t}^{C}$ are extracted from the same data set by analytic continuation of the TAP's for the ${ }^{4} \mathrm{He}\left(\mathrm{d},{ }^{3} \mathrm{He}\right)^{3} \mathrm{H}$ reaction to the proton-exchange pole $\left(\eta_{t}^{C}\right)$ and neutron-exchange pole $\left(\eta_{t}\right)$. The results quoted involve averages over all incident energies (5 values). Emphasis is placed on careful handling of the polynomial expansions, in particular, the Coulomb singularities, with an aim to minimize any truncation errors in the analytic continuation process (see discussion above for extraction of $\eta_{d}$ ). Within the context of a model for the ${ }^{3} \mathrm{He} \rightarrow \mathrm{p}+\mathrm{d}$ vertex, the two results from the transfer reactions for $\eta_{t}^{C}$ are consistent with each other and larger in magnitude than the result from the $\mathrm{AC}$ analysis. The latest value, within errors, is completely consistent with the $\mathrm{AC}$ result, whereas the earlier value differs by more than one standard deviation.

By comparing the results given in Tables 1 and 2, we can see that the three latest theoretical values for $\eta_{t}$ are consistent with the latest experimental results. In contrast, the work of Friar et al. $/ 39 /$, in which Coulomb effects were treated fully for $\eta_{t}^{C}$ $/ 13,39 /$, shows that $\eta_{t}^{C}$ is expected to be only $\sim 6.5 \%$ smaller in magnitude than $\eta_{t}$; whereas, the experimental $\eta_{t}^{C}$ is determined from the AC method to be $30 \%$ smaller in magnitude than $\eta_{t}$. On the other hand, within the limitations of the extraction, the transfer reaction results are comparable to the theoretical prediction. Theory and experiment agree upon the direction of the Coulomb effect, but disagree on the magnitude of the effect. One aspect that can be explored theoretically is whether charge symmetry breaking effects in the NN interaction alter significantly the theoretical values for $\eta_{t}^{C}$. Currently, 
theory indicates that $\eta_{t} / \eta_{t}^{C}=1.07 \pm 0.04$, whereas the $\mathrm{AC}$ method values give $\eta_{t} / \eta_{t}^{C}=1.46 \pm 0.24$. Coulomb effects, according to theory, are negligible for $\widetilde{D}_{2}$ because of the compensating effects of $F(\kappa)$ and $\mu^{C}<\mu$ in Eq. (11). To the extent that $\widetilde{D}_{2}$ is a good approximation to $D_{2}$, we would expect that $D_{2}^{t} \simeq D_{2}^{t C}$ as well. Besides the indication given above that $\widetilde{D}_{2}$ overestimates $D_{2}$ by about $5 \%$ in magnitude, there is further theoretical indication that it may be as great as 8 or $9 \% / 42 /$. However, as the reader can see, the experimental data for $D_{2}$ are not precise enough to distinguish such subtleties. In fact, all theoretical values for $D_{2}$, or its approximation through $\eta$, are fully consistent with the latest experimental results.

At the end of the theory section directly above, we mentioned that there were empirical arguments for choosing the coupling convention such that the signs of $\eta$ and $D_{2}$ for the three-nucleon systems were opposite to that for the deuteron. This evidence comes from comparing the $(d, p)$ and $\left(d,{ }^{3} H\right)$ TAP's for the two cases. As background, consider a single-nucleon exchange Feynman diagram as given in Fig. 1.

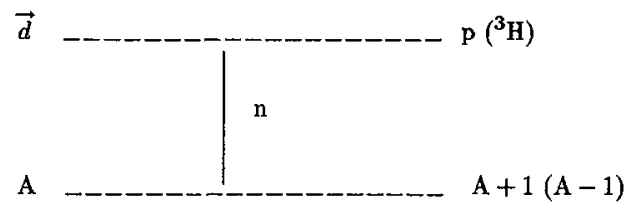

Fig. 1 - Feynman diagram for $(d, p)$ or $\left(d,{ }^{3} H\right)$ transfer reactions.

Evaluation of such a diagram corresponds to the plane-wave approximation within the context of distorted-wave theory. Using the momentum-space version of Eq. (4) to construct the upper vertex and a similar, but generalized form for the lower vertex, one can write down the Feynman amplitude /62/ and derive the TAP's. Because the exchanged particle is spin $1 / 2$, the cross section factors into the contribution from the upper vertex times the contribution from the lower vertex. This also occurs for the cross section with the deuteron polarized. As a consequence, the TAP's (Madison convention) depend only on the ratio of the D-wave momentum-distribution amplitude to the S-wave momentum-distribution amplitude here called $\Delta=f_{2}\left(\mathrm{P}_{r}\right) / f_{0}\left(\mathrm{p}_{r}\right)$ :

$$
\mathrm{T}_{2 q}=2 \frac{\Delta\{1+\Delta / 2 \sqrt{2}\}}{1+\Delta^{2}} \sqrt{\frac{4 \pi}{5}} \mathrm{Y}_{q}^{[2]}\left(\hat{\mathrm{p}}_{\mathrm{r}}\right)
$$

where $\vec{p}_{r}$ equals $-\vec{p}_{d} / 2+\vec{p}_{p}$ for $(\mathrm{d}, \mathrm{p})$ and $\overrightarrow{\mathrm{p}}_{d}-2 \overrightarrow{\mathrm{p}}_{H} / 3$ for $\left(\mathrm{d},{ }^{3} \mathrm{H}\right)$. I assumed that particle $\mathrm{x}$ is the deuteron and $\mathrm{y}$ the neutron for $\left(d,{ }^{3} H\right)$. This equation is not adequate for explaining the observed TAP's in transfer reactions, but it does make it possible to illustrate the points I wish to make about the signs. Moreover, with some information from the DWBA amplitude, it is possible to modify Eq. (12) such that a qualitative understanding of the TAP's can be had. On the other hand, Eq. (12) is precisely the equation that is used in the $\mathrm{AC}$ method $/ 53 /$.

Let us look at $\Delta$ in Eq. (12). The key to $\Delta$ is the $f_{l}\left(p_{r}\right)$. If we make an expansion of the $f_{l}\left(p_{r}\right)$ about $p_{r}=0$, then $\Delta$ can be expressed in terms of the DWBA parameter defined in Eq. (10):

$$
\Delta \simeq D_{2} \mathrm{p}_{r}^{2}+\mathrm{O}\left(\mathrm{p}_{\mathrm{r}}^{4}\right)
$$

Such an expansion has a radius of convergence $\left|\mathrm{p}_{r}\right|<\mu$ due to the nearest singularity of the $f_{l}\left(\mathrm{p}_{r}\right)$ which is a pole at $\mathrm{p}_{r}=\mathrm{i} \mu$. In fact, Laurent expansions of the $f_{l}\left(\mathrm{P}_{r}\right)$ about $\mathrm{P}_{r}=\mathrm{i} \mu$ permit us to obtain an expansion for $\Delta$ that has a radius of convergence around this point that is constrained by the nearest branch points of the $f_{l}\left(p_{r}\right)$. Specifically, we have

$$
f_{0}\left(\mathrm{p}_{r}\right)=\frac{\sqrt{\mu}}{\pi} \frac{C_{0}}{\mathrm{p}_{r}^{2}+\mu^{2}}+\sum_{n=0}^{\infty} \mathrm{a}_{n}^{(0)}\left(\mathrm{p}_{r}^{2}+\mu^{2}\right)^{n}
$$

and

$$
f_{2}\left(\mathrm{p}_{r}\right)=\frac{1}{\pi \sqrt{\mu^{3}}} \frac{C_{2} \mathrm{p}_{r}^{2}}{\mathrm{p}_{r}^{2}+\mu^{2}}+\mathrm{p}_{r}^{2} \sum_{n=0}^{\infty} \mathrm{a}_{n}^{(2)}\left(\mathrm{p}_{r}^{2}+\mu^{2}\right)^{n}
$$

With the aid of Eqs. (14) and (15), we can write an expansion for $\Delta$ about $p_{r}^{2}=-\mu^{2}$ since $\Delta$ is analytic at that point. The expansion has the form

$$
\Delta=-\frac{C_{2}}{C_{0}}+\sum_{n=1}^{\infty} \mathrm{b}_{n}\left(\mathrm{p}_{r}^{2}+\mu^{2}\right)^{n}
$$

Equation (16) makes clear how the TAP's are directly related to the $\eta$ 's by analytic continuation to the poles of the $f_{l}\left(\mathrm{p}_{r}\right)$ at $p_{r}^{2}=-\mu^{2}$. This is the basis of the AC method discussed above /53/. Furthermore, Eqs. (12) and (16) can be used to argue from the transfer-reaction data the different signs for $\eta$ in going from ${ }^{2} \mathrm{H}$ to ${ }^{3} \mathrm{H}$ and ${ }^{3} \mathrm{He}$. Let us see how this occurs.

Some years ago, Knutsen et al. /63,64/, starting from the full DWBA amplitude for the transfer reaction (d,p), presented a simplified picture of the TAP's that led to Eq. (12) except for two features: 1. The above unit vector in the spherical harmonic is replaced by the unit vector that points to the turning point of the classical Coulomb trajectory; 2 . $\Delta$ is evaluated at $\mathrm{p}_{r}=\mathrm{i} 3 \beta / 4$, where $\beta$ is the wave number for the binding of the neutron at the target vertex. In their most 
essential terms, these results come about because the neutron is assumed to be transferred at the classical turning point and for subCoulomb interactions this is at a great enough distance that only the asymptotic form of the neutron wave function comes into play. For a $(d, p)$ reaction on ${ }^{208} \mathrm{~Pb}$, it is easy to use the kinematics to show that the spherical harmonic in Eq. (12) behaves similarly whether it has as it argument the unit vector pointing to the turning point of the classical Coulomb trajectory or $\hat{\mathrm{p}}_{r}$. In addition, $3 \beta / 4=0.2268 \mathrm{fm}^{-1}$ for the transition leading to the positive parity, spin $1 / 2$, state at 2.032 $\mathrm{MeV}$ in ${ }^{209} \mathrm{~Pb} / 65 /$. Meanwhile, for ${ }^{2} \mathrm{H}, \mu=0.2316 \mathrm{fm}^{-1}$. Within this qualitative framework, one can see that the sign of the TAP's is determined by $\eta_{d}$, since the summation term in Eq. (16) essentially vanishes; moreover, we actually attain a rough description of the data.. Extending these arguments to the $\left(d,{ }^{3} H\right)$ reaction, we have a similar qualitative conclusion. Therefore, the sign of $\eta_{t}$ is determined from the TAP's given an initial angular-momentum coupling order. What is the nature of the data? An example can be seen in Fig. 2, the measured TAP's have essentially opposite-sign behavior, indicating that one should work within a framework that indicates this fact: Since the essential parameter controlling the sign of the TAP's is $\eta$, it seems appropriate to choose the coupling for ${ }^{3} \mathrm{H} \rightarrow \mathbf{n}+\mathrm{d}$ such that $\eta_{t}$ has a negative sign, remembering that there is no freedom to choose the sign of $\eta_{d}$ and that the experimental TAP's lead to $\eta_{d}>0$. Thus, that is the basis for adopting the coupling convention indicated above for ${ }^{3} \mathrm{H} \rightarrow \mathrm{n}+\mathrm{d}$.

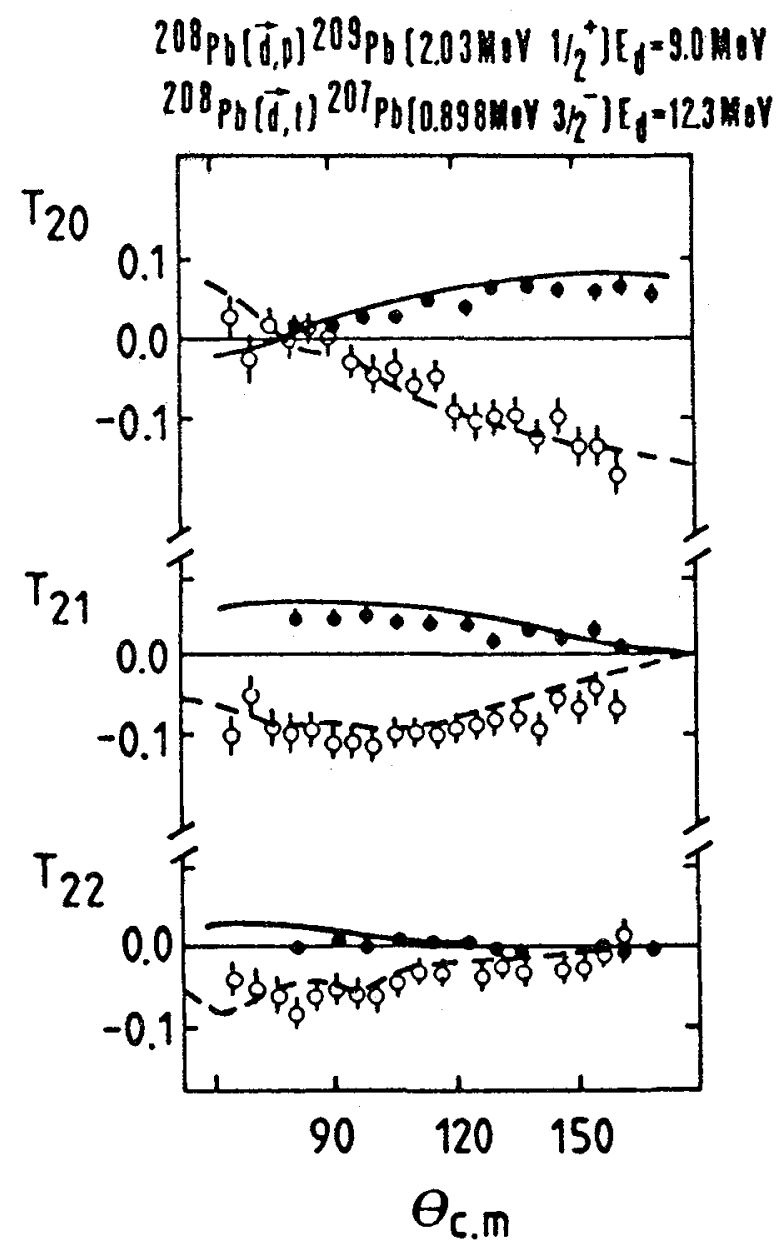

Fig. 2 - Comparison of the TAP angular distributions for $(d, p)$ [full circles] and $\left(d,{ }^{3} H\right)$ [open circles] transfer reactions /11/. The data are from $/ 66 /$. The curves are LEA DWBA calculations that include the $S$ - and D-wave components at the fewnucleon vertex. The full lines are for $(d, p)$ and the dashed lines are from $\left(d,{ }^{3} \mathrm{H}\right)$.

${ }^{4} \mathrm{He}:$

Theory

A D-state presence in ${ }^{4} \mathrm{He}\left(0^{+}\right)$can be asymptotically realized between two bound-state fragments only for two deuterons 
with relative orbital-angular momentum of 2 and total spin-angular momentum of 2 coupled to 0 . To date, an experimental determination of $\eta_{\alpha}$ for this configuration by means of dispersion-relation methods or pole extrapolation with appropriate TAP data has not been carried out. The main empirical results come from the TAP's of (d, $\alpha$ ) transfer reactions, where attempts are made to extract $D_{2}^{\alpha}$ for ${ }^{4} \mathrm{He} \rightarrow \mathrm{d}+\mathrm{d}$.

What does theory say about $D_{2}^{\alpha}$ ? Though there have been several four-body calculations for ${ }^{4} \mathrm{He}$ including D-states $/ 45,48,67-72 /$, only three $/ 45,48,67 /$ report $D_{2}^{\alpha}$ values and none give $\eta_{\alpha}$. Using the Amalgamation of Two-body correlations into Multiple Scattering processes (ATMS) ${ }^{4}$ He wave function from the Reid-Soft-Core (RSC) NN interaction /30/ of Akaishi $/ 73 /$, modified to have Hankel-function asymptotic behavior with correct separation energy between the two deuteron clusters, Tostevin $/ 67 /$ gets $D_{2}^{\alpha}=-0.08 \mathrm{fm}^{2}$. Schiavilla et al. /45/ obtain ${ }^{4} \mathrm{He}$ variational (Monte Carlo) wave functions with the Urbana /47/ and Argonne /46/ NN interactions augmented by a phenomenological three-nucleon interaction that is adjusted to give good values for the binding energies of the $\mathrm{A}=3$ and $\mathrm{A}=4$ nuclei. From these models, $D_{2}^{\alpha}=-0.24 \mathrm{fm}^{2}$ (Urbana) and $-0.16 \mathrm{fm}^{2}$ (Argonne) where the binding energies are $-28.2 \pm 0.4$ and $-27.8 \pm 0.4 \mathrm{MeV}$, respectively. These theoretical results indicate that $D_{2}^{\alpha}$ is negative, but reach no consensus on it magnitude.

Most recently, Morita et al. /48/ have also calculated $D_{2}^{\alpha}$ for several different NN interactions. Their thesis is that since ${ }^{4} \mathrm{He}$ is the most tightly bound few-body system, we might expect differences in representing the short-range behavior of the NN forces to show up in certain observables. In particular, the $D_{2}^{\alpha}$ is shown to be extremely sensitive to the short-range behavior of the tensor component of the NN force by using ATMS generated four-body wave functions. Their idea traces back to the original suggestion by Santos and Eiró $/ 74 /$, but the latter authors were nó able to check it with wave functions derived from realistic NN interactions. With three different $N N$ interactions, $\mathrm{RSCV}_{8} / 47 /, \mathrm{AV}_{14} / 46 /$, and the TRS $/ 49 /$, they find $D_{2}^{\alpha}=-0.12,-0.21$, and $-0.27 \mathrm{fm}^{2}$, respectively. The first thing that we note is that the RSC result is close to that of Tostevin given above (remember the modifications Tostevin made to the tail of the wave function). Secondly, we see large differences among their three results. Morita et al. argue that these differences have their source in the strength of the tensor force, i.e., in moving from TRS $\rightarrow \mathrm{AV} \rightarrow \mathrm{RSC}$, the depth of the tensor-force potential becomes deeper and deeper. The deeper the tensor force well, the more strongly it pulls the effective d-d wave function, $U_{2}(\rho)$, in ${ }^{4} \mathrm{He}$ towards $\rho=0$. As a consequence, the $\rho^{4}$ in the numerator integrand for $D_{2}^{\alpha}$ [Eq. (10)] suppresses the D-wave contribution. They suggest that there might be a relationship between $D_{2}^{\alpha}$ and the depth of the tensor component of the NN interaction. However, what they do not tell us is the binding energy for ${ }^{4} \mathrm{He}$ in the three cases. In one of their earlier papers $/ 75 /$, the value $-21.9 \mathrm{MeV}$ is given for the RSC interaction. However, since binding energy plays a large role in the D-state asymptotic parameters, it is essential to know if their is a correlation. Nevertheless, we should note that they find no corresponding sensitivity to the interaction for $D_{2}^{t}$ as can be seen in Table 1 where the value remains fixed for the three interactions at $-0.21 \mathrm{fm}^{2}$.

\section{Experiment}

Though no quantitative information has been extracted for $\eta_{\alpha}$ by analyticity methods, the first qualitative empirical evidence for a D-state component in the ${ }^{4} \mathrm{He}$ ground state was obtained by Plattner et al. $/ 76 /$, who applied a forward-angle dispersion relation (FDR) to elastic $\mathrm{p}+{ }^{4} \mathrm{He}$ scattering. In principle, FDR's can be extrapolated to negative energies to extract asymptotic normalization constants from the residues at poles corresponding to bound states of the external fragments. For example, this might be thought to be a possibility for elastic $d+d$ scattering, though the ${ }^{4} \mathrm{He}$ bound state is far removed from the physical scattering energies.

Despite the lack of an experimental determination of $\eta_{\alpha}$, progress has been made in showing that $D_{2}^{\alpha}$ is nonzero and negative from investigations of $(\mathrm{d}, \alpha)$ transfer reactions $/ 77,78 /$. However, there does not exist a consensus on the magnitude to date. The two references just quoted give as values $-0.3 \pm 0.1$ and $-0.19 \pm 0.04 \mathrm{fm}^{2}$, respectively. Unlike the $(\mathrm{d}, \mathrm{p})$ and $\left(\mathrm{d},{ }^{3} \mathrm{H}\right)$ transfer reactions where a single nucleon is exchanged, the $(d, \alpha)$ transfer reaction involves the transfer of a pair of nucleons or in the simplest assumption a deuteron. The complications of the exchange of a spin-1 object show up already in looking at a simple Feynman diagram like Fig. 1 with the exchanged neutron replaced by a deuteron and the vertices changed appropriately. By carrying out the algebra, it is clear that the cross section does not factorize into a product of the uppervertex quantities with the lower-vertex quantities. One gets interference effects from the S- and D-wave contribution of the $\alpha$ vertex with those of the target vertex. On the other hand, this complication may lead to large sensitivities to these components. A detailed commentary of these aspects can be found in Refs. $/ 10,11$. Suffice it to say that, currently, theory and experiment agree on the sign of $D_{2}^{\alpha}$, but neither comes to any definite conclusions about its magnitude.

\section{4 - SHAPES OF THE 's-SHELL' NUCLEI}

Knowledge of the signs of $\eta$ for the s-shell nuclei permit us to make conclusions about their shapes even though only ${ }^{2} \Pi$ has a quadrupole moment /11/. The reasoning $I$ use in each case begins from Eq. (4) and utilizes the asymptotic forms given in Eq. (5). The important aspect is the D-wave component in Eq. (4). Let us begin with the deuteron.

For the deuteron, there are four configurations that we shall consider (the z-axis is assumed to be the quantization axis): nucleon spins aligned along the z-axis with $\rho$ either parallel or perpendicular to the z-axis and opposing nucleon spins (proton spin parallel to z-axis) with $\rho$ either parallel or perpendicular to the z-axis. For these four configurations, respectively, we find that Eq. (4) gives for large $\rho$ the following: $\left(1+\eta_{d} / \sqrt{2}\right),\left(1-\eta_{d} / 2 \sqrt{2}\right),\left(1-\sqrt{2} \eta_{d}\right) / \sqrt{2}$, and $\left(1+\eta_{d} / \sqrt{2}\right) / \sqrt{2}$, where I have dropped all common factors. For $\eta_{d}>0$, the first configuration has the greatest probability amplitude. This implies elongation along the spin quantization axis consistent with the prolate shape expected on the basis of $\mathrm{Q}_{d}>0$. It should be noted that I considered all possible different spin projections. For example, nucleon spins antialigned along the z-axis leads to nothing new. Also, it is interesting to observe that for these four configurations, the tensor operator $S_{12}$ has the values $+2,-1,-2$, and +1 , respectively $/ 79 /$, indicating that the coupling of the $d$-wave component to the s-wave component in the deuteron is strongest, i.e., most attractive, for the first configuration, consistent, again with $Q_{d}>0$. 
Proceeding onto ${ }^{3} \mathrm{H}$ and ${ }^{3} \mathrm{He}$, there are again four different asymptotic configurations to consider. Within the adopted convention that particle $\mathrm{x}$ is the deuteron and $\mathrm{y}$ is the nucleon, they are as follows: $\mathrm{m}_{d}=1, \mathrm{~m}_{N}=-1 / 2$ with $\rho$ either parallel or perpendicular to the z-axis and $\mathrm{m}_{d}=0, \mathrm{~m}_{N}=1 / 2$ with $\rho$ either parallel or perpendicular to the z-axis. For these configurations, in order, we get from Eq. (4) asymptotically the following: $\sqrt{2}\left(1+\eta_{t} / \sqrt{2}\right) / \sqrt{3}, \sqrt{2}\left(1-\eta_{t} / 2 \sqrt{2}\right) / \sqrt{3}$, $\left(1-\sqrt{2} \eta_{t}\right) / \sqrt{3}$, and $\left(1+\eta_{t} / \sqrt{2}\right) / \sqrt{3}$. Now we know from the empirical and theoretical results above that $\eta_{t}<0$. Therefore, the second configuration has the greatest probability amplitude. Combined with the amplitude for the first configuration (which is destructive between the $\mathrm{S}$-wave contribution and the $\mathrm{D}$-wave contribution), I conclude that ${ }^{3} \mathrm{H}$ is flattened in the $\mathrm{z}$ direction and stretched in the $\mathrm{x}-\mathrm{y}$ directions, i.e., ${ }^{3} \mathrm{H}$ has an oblate shape. Similarly for ${ }^{3} \mathrm{He}$. Between the spectator nucleon and the unlike nucleon within the deuteron, the tensor operator $S_{12}$ has the values $-2,+1,+2$, and -1 , respectively, for these configurations. It is interesting that the configuration with the largest $S_{12}$ does not have the largest asymptotic probability amplitude. However, the largest probability amplitude is associated with a positive $S_{12}$, that is, an attractive coupling of the tensor force.

Finally, for ${ }^{4} \mathrm{He}$, we have two identical, indistinguishable, deuterons. We have two indistinguishable configurations which have $\mathrm{m}_{d}^{1}=1, \mathrm{~m}_{d}^{2}=-1$ or $\mathrm{m}_{d}^{1}=-1, \mathrm{~m}_{d}^{2}=1$ and a third configuration where $\mathrm{m}_{d}^{1}=\mathrm{m}_{d}^{2}=0$. We can do one of the indistinguishable amplitudes an remember that its probability will be multiplied by 2 . Again, for each case, we take $\rho$ parallel and perpendicular to the z-axis. The results are, in order, the following: $\left(1+\eta_{\alpha} / \sqrt{2}\right) / \sqrt{3},\left(1-\eta_{\alpha} / 2 \sqrt{2}\right) / \sqrt{3}$, $-\left(1-\sqrt{2} \eta_{\alpha}\right) / \sqrt{3}$, and $-\left(1+\eta_{\alpha} / \sqrt{2}\right) / \sqrt{3}$. Since experiment tells us that $D_{2}^{\alpha}<0$, we know that $\eta_{\alpha}<0$ also. Therefore, remembering that the probability of the first two amplitudes must be counted twice, the largest contribution comes from the second amplitude with the first amplitude being destructive in its S-D interference. So, just like ${ }^{3} \mathrm{H}$ or ${ }^{3} \mathrm{He}$, ${ }^{4} \mathrm{He}$ has an oblate shape. Again, it should be noted that, between the unlike nucleons across clusters, $\mathrm{S}_{12}$ has the values $-2,+1,+2$. and -1 , respectively.

\section{5 - MOVING OUT OF THE 'S-SHELL' $={ }^{6} \underline{\mathrm{Li}}$}

In principle, D-sate asymptotic normalization constants exist for two asymptotic configurations of ${ }^{6} \mathrm{Li}$ : $\alpha \mathrm{d}$ and ${ }^{3} \mathrm{H}-{ }^{3} \mathrm{He}$. So far, experimental and theoretical efforts have focused solely on the $\alpha$ d configuration.

Theoretical work is so far limited. There has been only one published calculation of $\eta_{\alpha d} / 80 /$ and $D_{2}^{\alpha d} / 81 /$ based on a three-body ( $\alpha \mathrm{np}$ ) model of ${ }^{6} \mathrm{Li}$. Such three-body models, generated from the underlying two-body interactions, $\alpha \mathrm{N}$ and $\mathrm{np}$, give a reasonable description of many ${ }^{6} \mathrm{Li}$ properties $/ 80 /$ and $\mathrm{d} \alpha$ scattering $/ 82 /$. Depending on whether the $\mathrm{S}_{1 / 2} \alpha \mathrm{N}$ interaction, that component of the $\alpha \mathrm{N}$ interaction that makes manifest the Pauli repulsion between the $\alpha$ and the other two nucleons, is represented by a purely repulsive potential or an attractive potential with the forbidden bound state removed, the three-body models predict that $\eta_{\alpha d}=0.00817$ or 0.00551 , respectively $/ 80 /$. For both cases the underlying np interaction leads to $4 \%$ deuteron $D$-state component. The corresponding $D_{2}^{\alpha d}$ values are 0.0852 and $0.0617 \mathrm{fm}^{2}$, respectively $/ 81 /$. These calculations have recently been extended /83/. Instead of the simple Yamaguchi-Yamaguchi interaction for the np pair, the Paris interaction is used by means of the PEST (rank-6) representation. Without changing the underlying $\alpha \mathrm{N}$ interactions, we obtain $\eta_{\alpha d}=0.0194$ and 0.0169 , respectively, and $D_{2}^{\alpha d}=0.226$ and $0.218 \mathrm{fm}^{2}$, respectively. The latter values represent an increase of more than a factor of 2 over the above results, while the binding energy of ${ }^{6} \mathrm{Li}$ decreased by only $\sim 6 \%$ for both $\alpha \mathrm{N}$ models. On the basis of attributing the full quadrupole moment of ${ }^{6} \mathrm{Li}$ to the alpha-deuteron component of the wave function, it has been argued that $\eta_{\alpha d}<0 / 84,11$, a clear conflict with the sign predicted with the three-body wave functions. The question is open whether one can represent the small negative quadrupole moment of ${ }^{6} \mathrm{Li}$ by an effective $\alpha \mathrm{d}$ model. Calculations within the three-body framework are underway to check that approximation /83/.

Experimentally, there has been only one attempt to extract $\eta_{\alpha d}$ directly. Bornand et al. /85/, by application of a FDR to elastic $\mathrm{d} \alpha$ scattering, have extracted values for both the $\mathrm{S}$ - and $\mathrm{D}$-wave asymptotic normalization constants for ${ }^{6} \mathrm{Li} \rightarrow \alpha+\mathrm{d}$. From these values, one obtains $\eta_{\alpha d}=0.005 \pm 0.017$. Unfortunately, this result does not permit determination of the sign. On the other hand, the above theoretical predictions fall within the experimental error.

Most recently, there has been an attempt to extract information about $\eta_{\alpha d}$ by an indirect method. Santos et al. $/ 86 /$, by considering the reaction ${ }^{6} \mathrm{Li}(\mathrm{d}, \alpha)^{4} \mathrm{He}$ with polarized deuterons, come to the conclusion that $\eta_{\alpha d}$ is negative and lies in the range $-0.015<\eta_{\alpha d}<-0.010$, based on a DWBA analysis of the low-energy TAP's along with the assumption that $\eta_{\alpha}=-0.2$. In order to reproduce roughly the observed large TAP's, the ${ }^{4} \mathrm{He}$ and ${ }^{6} \mathrm{Li}$ D-state components must interfere constructively in the asymptotic region. This implies that they have the same sign. One might take this result as the first direct evidence of the small $\mathrm{D}$-state component in ${ }^{6} \mathrm{Li} \rightarrow \alpha+\mathrm{d}$.

\section{6 - D-STATES AND THE ROLE OF RADIATIVE CAPTURE REACTIONS}

Radiative capture reactions with polarized deuterons have produced beautiful empirical evidence for the D-state in ${ }^{3} \mathrm{He} / 87$ $89 /$ and ${ }^{4} \mathrm{He} / 90-92 /$, and possibly in the future will do the same for ${ }^{6} \mathrm{Li}$ through ${ }^{4} \mathrm{He}(\mathrm{d}, \gamma)^{6} \mathrm{Li} / 93 /$. Though the TAP's for ${ }^{1} \mathrm{H}(\mathrm{d}, \gamma){ }^{3} \mathrm{He}$ and ${ }^{2} \mathrm{H}(\mathrm{d}, \gamma){ }^{4} \mathrm{He}$ indicate directly that the D-states of ${ }^{3} \mathrm{He}$ and ${ }^{4} \mathrm{He}$ are primarily responsible for their nature, extracting quantitative information on the asymptotic observables, $\eta$ or $D_{2}$, is difficult. This is the case because it is essential to understand the reaction mechanism, i.e., which electromagnetic multipoles are coming into play, and it is necessary to understand how important it is to treat the initial-state rescattering exactly, i.e., with full three- or four-body dynamics. Except for one calculation in the case of ${ }^{3} \mathrm{He} / 88 /$, and new work underway for ${ }^{3} \mathrm{He} / 94 /$, all analyses for both ${ }^{3} \mathrm{He}$ and ${ }^{4} \mathrm{He}$ have been carried out within the framework of effective two-body dynamics $/ 10,11 \%$. One of the aims of our current work $/ 94 /$ is to assess the validity of the effective two-body models in interpreting the ${ }^{1} \mathrm{H}(\mathrm{d}, \gamma)^{3} \mathrm{He}$ data, especially the TAP's.

The first measurement of $T_{20}(\theta)$ for the ${ }^{1} \mathrm{H}(\mathrm{d}, \gamma)^{3} \mathrm{He}$ reaction was obtained at $E_{d}=19.8 \mathrm{MeV} / 87 /$. At this energy, the 
reaction is primarily an $\mathrm{E} 1$ transition, so in the absence of a D-state component in the ${ }^{3} \mathrm{He}$ wave function, $T_{20}$ would be zero. Thus, the observed, definitely nonzero, angular distribution for $T_{20}$ is direct evidence for the D-state in ${ }^{3}$ He. These data are described reasonably well with an effective two-body model generated from projections of three-body wave functions onto the Nd space. Furthermore, a value of $\eta_{t}^{C}=-0.035 \pm 0.010$ is extracted from the $T_{20}$ by using asymptotic wave functions (Coulomb neglected), Eq. (5), with an inner cutoff radius of $2.5 \mathrm{fm}$. Assumption of any reasonable behavior within $2.5 \mathrm{fm}$, except the asymptotic form, leads to no change in this value. This value is consistent with all the values given in Table 2 .

The Cartesian TAP $A y y\left(90^{\circ}\right)$ has been measured at $E_{d}=29.2$ and $45.3 \mathrm{MeV}$ with the results $0.0282 \pm 0.0016$ and $0.0113 \pm 0.0014$, respectively $/ 88 /$. These results were compared to a full three-body Faddeev calculation, bound and continuum, with the RSC potential. As expected, it is found that the calculation is dominated by the E1 transition. In addition, $89 \%$ of the contribution to $A_{y y}$ is due to capture into the trinucleon D-state component that is constructed from a pair of nucleons in a relative $\mathbf{P}$-wave with the remaining nucleon in a $\mathbf{P}$-wave relative to the pair's center-of mass. This particular component makes up $\sim 45 \%$ of the total D-state component of the wave function generated. The D-state of the deuteron has only a small effect on $A y y$, but initial-state rescattering, relative to a plane-wave treatment, leads to large corrections. The calculated $A_{y y}$ is $\sim 20 \%$ larger than the data points, indicating, perhaps, that the D-state generated by the RSC interaction is too large. Our preliminary results /94/, also in an exact Faddeev calculation with only the El operator, agree that the role of the deuteron D-state is almost negligible. Moreover, in the absence of P-wave interactions, we find that $A y y$ plotted as a function of energy changes sign just before $45 \mathrm{MeV}$. Thus, this may be an indication of an important role for $\mathrm{P}$-waves. This is interesting since $\mathrm{P}$-wave contribution to the $\mathrm{D}$-state in ${ }^{3} \mathrm{He}$ is not present in effective two-body models. Moreover, Ballot and Eiro /95/ have found this contribution to be only $\sim 12 \%$ in a hyperspherical calculation that involved an effective p-d potential to generate the initial-state rescattering. Clearly, much theoretical work needs to be done to more fully interpret experimental results. There also exist data at higher energy /89/ which require attention as well.

In light of the above discussion concerning ${ }^{3} \mathrm{He}$, it comes as no surprise that the theoretical understanding in the case of ${ }^{4} \mathrm{He}$ is more primitive. Nevertheless, the experimental effort has provided clear empirical evidence for the D-state in ${ }^{4} \mathrm{He} / 90 /$. The reaction ${ }^{2} \mathrm{H}(\mathrm{d}, \gamma)^{4} \mathrm{He}$ at low energies is dominated by the E2 transition (isospin considerations) and, in a simple analysis, one concludes that the TAP's vanish without a D-state component in the ${ }^{4} \mathrm{He}$ ground state (for pure E2). Interestingly, at $E_{d}=9.7 \mathrm{MeV}, T_{20}(\theta)$ is isotropic within experimental error and has the value $-0.22 \pm 0.014$. Other data now exist /91,92/ that provide considerable challenge to interpretation $/ 10,11 \%$. In addition, the very low-energy regime of relevance in astrophysical applications is of interest since the D-state of ${ }^{4} \mathrm{He}$ is expected to play a major role, but space limitations precludes my going into any detail ( please see Refs. /10,11/).

\section{7 - SUMMARY}

Our understanding of the essential ingredients involved in the generation of $\eta_{d}$ is on solid ground. We should now be in a position to try to generate from this knowledge a theoretical value for $\eta_{d}$ with accepted error limitations. In addition, we are at a stage where it is worthwhile to invest effort into understanding more deeply the role that subnucleon degrees of freedom may play in determining $\eta_{d^{*}}$. On the experimental side, conflicting results by two different methods (transfer reactions and $\mathrm{AC}$ ), each claiming very careful empirical analysis, are available. These need to be reconciled, perhaps by another careful look at the theoretical underpinnings of each extraction method. The $\eta_{d}$ result based on the np phase-shift analysis should be qualified with a careful look at possible systematic errors. Effort in the cases of ${ }^{3} \mathrm{H}$ and ${ }^{3} \mathrm{He}$ would now be well spent in trying to obtain as deep an understanding as possible of the relationship of $\eta_{t}$ and $\eta_{t}^{C}$ to $\eta_{d}$ and the underlying tensor force. Computationally, we are currently at the state of the art given an underlying NN plus $3 \mathrm{~N}$ interaction. Perhaps all that is really needed to understand $\eta_{t}$ or $\eta_{t}^{C}$ is the three-nucleon binding energy and $\eta_{d}$. On the other hand, the apparent relationship between $\eta_{t}$ and $\eta_{d}$ is not necessarily exact and may provide us other information about the tensor interaction. Experimentally, the AC method seems to have yielded an interesting picture with regard to possible differences between $\eta_{t}$ and $\eta_{t}^{C}$. However, it is necessary to have results of higher accuracy from either the AC method or the transfer reaction method to really test the theoretical models. Theoretical work should be pursued to determine if such large chargesymmetry-breaking effects can be generated for the $\eta$ 's in the three-body system. In addition, theoretical work should continue in the direction of exact three-body calculations to better understand the very nice data for the TAP's of radiative capture reactions. Unfortunately, such calculations are extremely difficult in the case of ${ }^{4} \mathrm{He}$, but would probably begin to solve most of the computational difficulties being experienced with effective two-body models. Nevertheless, calculation of simply $\eta_{\alpha}$ from an exact four-body wave function derived from realistic interactions would be valuable. Much effort is needed to pin down an accepted value for either $\eta_{\alpha}$ or $D_{2}^{\alpha}$, both theoretically and experimentally, but especially experimentally. In the case of ${ }^{6} \mathrm{Li}$, the field is wide open experimentally, and there is plenty of room for theoretical effort, both in the $\alpha \mathrm{d}$ channel and the ${ }^{3} \mathrm{H}-{ }^{3} \mathrm{He}$ channel.

\section{ACKNOWLEDGEMENTS}

I would like to thank Ana Eiro, Ben Gibson, Nate Rodning, and Henry Weller for correspondence and discussions concerning particular points of the above material. The work of the author is supported in part by the U. S. Department of Energy under grant No. DE-FG05-86-ER40270.

\section{REFERENCES}

/1/ Kellog, J.M.B., Rabi, I.I., and Zacharais, J.R., Phys. Rev. $\underline{55(1939) 381 . ~}$

/2/ Schwinger, J., Phys. Rev. 55 (1939)235.

/3/ Amado, R.D., Phys. Rev. C19(1979)1473; Comments Nucl. Part. Phys. 10(1981)131.

/4/ Friar, J.L., Phys. Rev. C20(1979)325.

/5/ Ericson, T.E.O. and Rosa-Clot, M., Ann. Rev. Nucl. Part. Sci. 35(1985)271; Nucl. Phys. A405(1983)497. 
/6/ Ballot, J.L., Eiró, A.M., and Robilotta, M.R., Phys. Rev. C뜨(1989)1459.

/7/ Bishop, D.M. and Cheung, L.M., Phys. Rev. A20(1979)381.

/8/ Gerjuoy, E. and Schwinger, J., Phys. Rev. 61(1941)138.

19/ For example, in the three-nucleon system, see Gibson, B.F. and Lehman, D.R., Phys. Rev. C29(1984)1017.

/10/ Weller, H.R. and Lehman, D.R., Ann. Rev. Nucl. Part. Sci. $\underline{38}(1988) 563$.

/11/ Eiró, A.M. and Santos, F.D., J. Phys. G: Nucl. Part. Phys. 16(1990) in press.

/12/ Johnson, R.C. and Santos, F.D., Part. Nucl. 2(1971)285; Knutson, L.D., Ann. Phys. 106(1977)1.

/13/ Friar, J.L., Gibson, B.F., Lehman, D.R., and Payne, G.L., Phys. Rev. C25(1982)1616.

/14/ Knutson, L.D. and Haeberli, W., Phys. Rev. Lett. 35(1975)558.

/15/ Klarsfeld, S., Martorell, J., and Sprung, D.W.L., J. Phys. G10(1984)165.

/16/ Klarsfeld, S., Martorell, J., and Sprung, D.W.L., J. Phys. G10(1984)L205.

/17/ Klarsfeld, S., Martorell, J., Oteo, J.A., Nishimura, M., and Sprung, D.W.L., Nucl. Phys. A456(1986)373.

/18/ Ericson, T.E.O. and Rosa-Clot, M., J. Phys. G10(1984)L201.

/19/ P. Kroll, in Physics Data (Fachinform-Zentrum, Karlsruhe, 1981), Chap. 22.1.

/20/ Rodning, N.L. and Knutson, L.D., Phys. Rev. C41(1990)898.

/21/ Ericson, T.E.O., Nucl. Phys. A416(1984)281c.

/22/ Friar, J.L., Gibson, B.F., and Payne, G.L., Phys. Rev. C30(1984)1084.

/23/ Guichon, P.A.M. and Miller, G.A., Phys. Lett. 134B(1984)15.

/24/ Sitarski, W.P., Blunden, P.G., and Lomon, E.L., Phys. Rev. C $\underline{36}(1987) 2479$.

/25/ Dymarz, R., Morningstar, C.J., Gowrishankar, R., and Khanna, F., Nucl. Phys. A507(1990)531.

/26/ Rodning, N.L. and Knutson, L.D., Ref. /20/ and Phys. Rev. Lett. $\underline{75}$ (1986)2248.

/27/ Borbély, I., Grüebler, W., Vuaridel, B., and König, V., Nucl. Phys. ㅅ503(1989)349.

/28/ Stoks, V.G.J., van Campen, P.C., Spit, W., and DeSwart, J.J., Phys. Rev. Lett. 60(1988)1932.

/29/ Goddard, R.P., Knutson, L.D., and Tostevin, J.A., Phys. Lett. 118B(1982)241.

/30/ Reid, R.V., Ann. Phys. $\underline{50}(1968) 411$.

/31/ Lacombe, M., Loiseau, B., Richard, J.M., Vinh Mau, R., Côté, J., Pirè, P., and deTourreil, R., Phys. Rev. $\mathrm{C} 2 \underline{1}(1980) 861$.

/32/ Machleidt, R., Holinde, K. and Elster, CH., Phys. Rep. 149(1987)1.

/33/ Londergan, J.T., Price, C.E., and Stephenson, E.J., Phys. Rev. C쪼(1987)902.

/34/ Santos, F.D. and Colby, P.C., Nucl. Phys. A367(1981)197.

/35/ Avakov, G.B., Blokhintzev, L.D., Mukhamedzhanov, A.M., and Yarmukhamedov, P., Yad. Fiz 43(1986)824; Sov. J. Nucl. Phys, $43(1986) 524$.

/36/ Friar, J.L., Gibson, B.F., and Payne, G.L., Phys. Rev. C36(1987)1138.

/37/ Lehman, D.R. and Gibson, B.F., Phys. Rev. C13(1976)35.

/38/ Kim, Y.E., Sander, C., and Tubis, A., Phys. Rev. C14(1976)2008.

/39/ Friar, J.L., Gibson, B.F., Lehman, D.R., and Payne, G.L., Phys. Rev. C37(1988)2859.

/40/ Klepacki, D.J., Kim, Y.E., and Brandenberg, R.A., Phys. Rev. Cㅉ8(1988)998.

/41/ Ishikawa, S. and Sasakawa, T., Phys. Rev. Lett. $\underline{56}(1986) 317$.

/42/ Ishikawa, S. and Sasakawa, T., Few-Body Systems 1(1986)143.

/43/ Benayoun, J.J., Gignoux, C., and Chauvin, J., Phys. Rev, C2ㄱ(1981)1854.

/44/ Kim, Y.E. and Muslim, Phys. Rev. Lett. $42(1979) 1328$.

/45/ Schiavilla, R., Pandharipande, V.R., and Wiringa, R.B., Nucl. Phys. A449(1986)219.

/46/ Wiringa, R.B., Smith, R.A., and Ainsworth, T.L., Phys. Rev. C29 (1984)1207.

/47/ Lagaris, I.E. and Pandharipande, V.R., Nucl. Phys. A359(1981)331.

/48/ Morita, H., Akaishi, Y., and Tanaka, H., Prog. Theor. Phys. 79(1988)1279L.

/49/ deTourreil, R., Rouben, B., and Sprung, S.W.L., Nucl. Phys. A242(1975)445.

/50/ Frederico, T., Adhikari, S.K., and Hussein, M.S., Phys. Rev. C37(1988)364.

/51/ Adhikari, S.K. and Frederico, T., "Analytical Model for the Triton Asymptotic D-State Parameters", manuscript, 1990.

/52/ Knutson, L.D., Hichwa, B.P., Barroso, A., Eiró, A.M., Santos, F.D., and Johnson, R.C., Phys. Rev. Lett. $\underline{35}(1975) 1570$.

/53/ Vuaridel, B., Grüebler, W., König, V., Elsener, K., Schmelzbach, P.S., Bittcher, M., Singy, D., Borbély, I., Bruno, M., Cannata, F., and D'Agostino, M., Nucl. Phys. A499(1989)429.

/54/ Borbély, I., Grüebler, W., König, V., Schmelzbach, P.A., and Jenny, B., Phys. Lett. 109B(1982)262.

/55/ Borbély, I., König, V., Grüebler, W., Jenny, B., and Schmelzbach, P.A., Nucl. Phys. A351(1981)107.

/56/ Bhat, C.M., Ludwig, E.J., Clegg, T.B., and Karwowski, H.J., PARIS90, contribution $42 B$.

/57/ Bhat, C.M., Clegg, T.B., Karwowski, H.J., and Ludwig, E.J., Phys. Rev. C37(1988)1358.

/58/ Merz, F., Clement, H., Eckle, F.J., Eckle, G., Eiró, A.M., Graw, G., Kader, H., Roman, S., Schiemenz, P., and Seichert, N., Phys. Lett. B183(1987)144.

/59/ Sen, S. and Knutson, L.D., Phys. Rev. C26 (1982)257, as an example.

/60/ Entezami, F., Brown, J.D., Barnwell, J.M., Dhuga, K.S., Karbon, O., Nelson, J.M., and Roman, S., Nucl. Phys. $\underline{\mathrm{A} 405}(1983) 69$.

/61/ Ioannides, A.A., Nagarajan, M.A., and Shyam, R., Phys. Lett. 103B(1981)187.

/62/ Shapiro, I.S., in Selected Topics in Nuclear Theory, edited by F. Janouch (International Atomic Energy Agency, Vienna, 1963), p. 85.

/63/ Knutson, L.D., Stephenson, E.J., and Haeberli, W., Phys. Rev. Lett. 32(1974)690.

/64/ Knutson, L.D., Ann. Phys. 106(1977)1.

/65/ Knutson, L.D. and Rodning, N.L., J. Phys. G:Nucl. Phys. 13(1987)L109.

/66/ Knutson, L.D., Thompson, J.A., and Reyer, H.O., Nucl. Phys. A241(1975)36. 
/67/ Tostevin, J.A., in Proc. Tsukuba Int. Workshop on Deuteron Involved Reactions and Polarization Phenomena, Tsukuba, Japan (World Scientific, Singapore, 1985), p241; J. Phys. G11(1985)L211.

/68/ Ballot, J.L., Phys. Lett. 127B(1983)399.

/69/ Goldhammer, P., Phys. Rev. C229(1984)1444.

/70/ Carlson, J., Phys. Rev. C.38(1988)1879; Nucl. Phys. A508(1990)141c.

/71/ Fonseca, A.C., Phys. Rev. C40(1989)1390; Nucl. Phys. A508(1990)281c.

/72/ Chiang, P.C., Meder, M.R., and Purcell, J.E., Phys. Lett. 235B(1990)225.

/73/ Akaishi, Y., Nucl. Phys. A416(1984)409c.

/74/ Santos, F.D. and Eiró, A.M., Portgal. Phys. 15(1984)65.

/75/ Morita, H., Akaishi, Y., Endo, O., and Tanaka, H., Prog. Theor. Phys. 78(1987)1117.

/76/ Plattner, G.R., Viollier, R.D., and Alder, K., Phys. Rev. Lett. $34(1975) 830$.

/77/ Karp, B.C., Ludwig, E.J., Bowsher, J.E., Burks, B.L., Clegg, T.B., Santos, F.D., and Eiró, A.M., Nucl. Phys. $\underline{\mathrm{A} 457}(\mathbf{1 9 8 6}) 15$.

/78/ Merz, F., Eiró, A.M., Eckle, F.J., Eckle, G., Graw, G., Hertenberger, R., Kader, H., and Schiemenz, P., Nucl. Phys. A489(1988)399.

/79/ Blatt, J.M. and Weisskopf, V.F., Theoretical Nuclear Physics (John Wiley, New York, 1952), p.98.

/80/ Lehman, D.R. and Rajan, M., Phys. Rev. C25(1982)2743; Parke, W.C. and Lehman, D.R., Phys. Rev. C229(1984)2319.

/81/ Lehman, D.R. and Parke, W.C., Phys. Rev. C31(1985)1920; C377(1988)E2266.

182/ Koike, Y., Prog. Theor, Phys. $\underline{59}$ (1978)87; Nucl. Phys. A301(1978)411; A337(1980)23.

/83/ Woloschek, J.P. and Lehman, D.R., work in progress.

/84/ Nishioka, H., Tostevin, J.A., and Johnson, R.C., Phys. Lett. B124(1983)17.

/85/ Bornand, M.P., Plattner, G.R., Viollier, R.D., and Alder, K., Nucl. Phys. A294(1978)492.

/86/ Santos, F.D., Thompson, I.J., and Eiró, A.M., PARIS90, contribution 73B.

/87/ Vetterli, M.C., Kuehner, J.A., Trudel, A.J., Woods, C.L., Dymarz, R., Pilt, A.A., and Weller, H.R., Phys. Rev. Lett. $\underline{54}(1985) 1129$.

/88/ Jourdan, J. Baumgartner, M., Burynski, E., Egelhof, P., Klein, A., Pickar, M.A., Plattner, G.R., Ramsay, W.D., Rofer, H.W., Sick, I., and Torre, J., Nucl. Phys. A453(1986) 220.

/89/ Pitts, W.K., Meyer, H.O., Bland, L.C., Brown, J.D., Byrd, R.C., Hugi, M., Karwowski, H.J., Schwandt, P., Sinha, A., Sowinski, J., and van Heerden, I.J., Phys. Rev C프(1988)1.

/90/ Weller, H.R., Colby, P., Roberson, N.R., and Tilley, D.R., Phys. Rev. Lett. $\underline{53}(1984) 1325$.

/91/ Mellema, S., Wang, T.R., and Haeberli, W., Phys. Lett. 166B(1986)282.

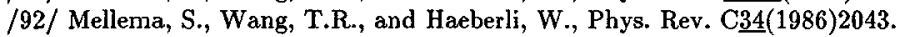

/93/ Weller, H.R., private communication.

194/ Fonseca, A.C. and Lehman, D.R., work in progress.

/95/ Ballot, J.L. and Eiró, A.M., Few-Body Systems Suppl. 2(1987)389. 